\title{
DÜBLIN
}

Technological University Dublin

ARROW@TU Dublin

2010-01-01

\section{Authentication of Biometric Features using Texture Coding for ID Cards}

Jonathan Blackledge

Technological University Dublin, jonathan.blackledge@tudublin.ie

Eugene Coyle

Technological University Dublin, Eugene.Coyle@tudublin.ie

Follow this and additional works at: https://arrow.tudublin.ie/engscheleart

Part of the Databases and Information Systems Commons, Medical Biomathematics and Biometrics Commons, Other Computer Engineering Commons, and the Secured Transactions Commons

\section{Recommended Citation}

Blackledge, Jonathan and Coyle, Eugene, "Authentication of Biometric Features using Texture Coding for ID Cards" (2010). Conference papers. 153.

https://arrow.tudublin.ie/engscheleart/153

This Conference Paper is brought to you for free and open access by the School of Electrical and Electronic Engineering at ARROW@TU Dublin. It has been accepted for inclusion in Conference papers by an authorized administrator of ARROW@TU Dublin. For more information, please contact arrow.admin@tudublin.ie, aisling.coyne@tudublin.ie,gerard.connolly@tudublin.ie.

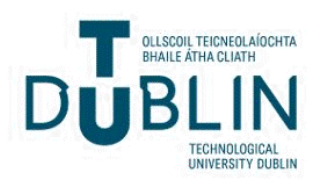




\title{
Authentication of Biometric Features using Texture Coding for ID Cards
}

\author{
J. M. Blackledge and E. Coyle \\ School of Electrical Engineering Systems, \\ College of Engineering and the Built Environment, \\ Dublin Institute of Technology, \\ Dublin, Ireland. \\ Email: jonathan.blackledge@dit.ie andeugene.coyle@dit.ie
}

\begin{abstract}
The use of image based information exchange has grown rapidly over the years in terms of both e-to-e image storage and transmission and in terms of maintaining paper documents in electronic form. Further, with the dramatic improvements in the quality of COTS (Commercial-Off-TheShelf) printing and scanning devices, the ability to counterfeit electronic and printed documents has become a widespread problem. Consequently, there has been an increasing demand to develop digital watermarking techniques which can be applied to both electronic and printed images (and documents) that can be authenticated, prevent unauthorized copying of their content and, in the case of printed documents, withstand abuse and degradation before and during scanning. In this paper we consider the background to a novel approach to solving this problem that has been developed into practically realisable system.
\end{abstract}

Keywords-Document Authentication, Texture Coding, Biometrics, ID Cards, Security and ID Documents

\section{INTRODUCTION}

In this paper, a new approach to digital watermarking is presented and a range of possible applications considered. The process is defined by using analytical techniques and concepts borrowed from Cryptography. It is based on computing a 'scramble image' by diffusing a 'watermark image' with a noise field (a cipher). For e-to-e applications, a cover image (covertext) can be introduced using a simple additive process ('confusion process'). The watermark is subsequently recovered by removing (subtracting) the covertext and then correlating the output with the original (key dependent) cipher. This approach provides the user with a method of hiding image-based information in a host image before transmission of the data. In this sense, the method provides a steganographic approach to transmitting encrypted information that is not apparent during an intercept. Decryption is based on knowledge of the key(s) and access to the host image.

With regard to digital image analysis and e-to-e communications, the method provides a way of embedding information in an image that can be used for authentication from an identifiable source, a method that is relatively insensitive to lossy compression, making it well suited to digital image transmission. However, with regard to doc- ument authentication, the use of diffusion and confusion using a covertext is not robust. The reason for this is that the registration of pixels associated with a covertext can not be assured when the composite image is printed and scanned. We therefore consider a diffusion only approach to document authentication which is robust to a wide variety of attacks including print/scan attacks, geometric, soiling and crumpling attacks. This is because the process of diffusion (i.e. the convolution of information) is compatible with the physical principles of an imaging system and the theory of image formation and thus, with image capture devices (digital cameras and scanners, for example) that, by default, conform to the 'physics' of optical image formation.

The diffusion of plaintext (in this case, an image) with a noise field (the cipher) has a synergy with the encryption of plaintext using a cipher and an XOR operation (when both the plaintext and cipher are represented by binary streams). However, decryption of a convolved image (deconvolution) is not as simple as XORing the ciphertext with the appropriate cipher. Here, we consider an approach which is based on pre-conditioning the original cipher in such a way that decryption (de-diffusion) can be undertaken by correlating the ciphertext with the cipher. The output ciphers generated for printed document authentication are textures of a type that are determined by the spectral characteristics of the plaintext which can be applied using low resolution COTS printers and scanners. In this sense, the approach is based on 'texture coding'. In this paper, we present a method of texture coding that has been developed into a practically vaibale system and present a range of example applications to which it has been applied. Examples of the robustnes of the system to various 'attacks' is provide in an extended Appendix.

\section{Transform Domain WATERMARKing Methods}

Like many aspects of digital signal and image processing, watermarking schemes fall into two categories: spatial domain and transform domain techniques [1], [2], [3], [4], [5]. This depends on whether the watermark is encoded by directly modifying pixels (such as simply flipping low-order bits of selected pixels) or by altering some frequency coeffi- 
cients obtained by transforming the image in the frequency domain. Spatial domain techniques are simple to implement and usually require a lower computational cost. However, such methods tend to be less robust to tampering than methods that place the watermark in the transform domain [6], [14], [15].

Watermarking schemes that operate in a transform space are increasingly common, as they posses a number of desirable features. These include the following: (i) By transforming spatial data into another domain, statistical independence between pixels and high-energy compaction is obtained; (ii) the watermark is irregularly distributed over the entire spatial image upon an inverse transformation, which makes it more difficult for attackers to extract and/or decode a watermark; (iii) it is possible to provide markers according to the perceptual significance of different transform domain components so that a watermark can be placed adaptively in an image where it is least noticeable, such as within a textured area [16], [17], [18], [19]. In addition, transform domain methods can hide information in significant areas of a covertext which makes them more robust to attacks and distortion while remaining visually imperceptible [20], [38], [22]. Cropping, for example, may seriously distort any spatially based watermark but is less likely to affect a transformbased scheme because watermarks applied in a transform domain are dispersed over the entire spatial domain so that upon inverse transformation, at least part of the watermark may be recovered. Lossy compression is an operation that usually eliminates perceptually unimportant components of an image and most processing of this type takes place in a transform domain. Thus, matching the transform with a compression transform can result in an improved performance (i.e. DCT for JPEG, Wavelet for JPEG-2000). Further, the characteristics of the Human Visual System (HVS) can be fully exploited in a transform domain, e.g. [23], [24].

With transform domain watermarking, the original host data is transformed to produce a matrix of 'coefficients'. These coefficients are then perturbed by a small amount in one of several possible ways in order to represent the watermark. Coefficient selection is based on 'perceptual significance' and/or 'energy significance'. When the watermarked image is compressed or modified by any image processing operation, noise is added to the already perturbed coefficients. Private retrieval operations involve subtracting the coefficients associated with the watermarked image from those of the original image to obtain the noise perturbation. The watermark is then estimated from the noisy data as best as possible. The most difficult problem associated with 'blind-mode' watermark detection (in which the host image is not available) in the frequency domain is to identify the coefficients used for watermarking. Embedding can be undertaken using quantization (thresholding) or image fusion, for example, but in either case, most algorithms consider the HVS to minimize perceptibility. The aim is to place as much information in the watermark as possible such that it is most robust to an attack but least noticeable. Most schemes operate directly on the components of some transform of the 'cover image' such as the Discrete Cosine Transform (DCT), Discrete Wavelet Transforms (DWT) and the Discrete Fourier Transform (DFT) [25], [26] [27].

In general, the HVS is not sensitive to small changes in edges and texture but very sensitive to small changes in the smoothness of an image [28], [29]. In 'flat' featureless portions of the image, information is associated with the lowest frequency components of the image spectrum, while, in a highly textured image, the information is concentrated in the high frequency components. The HVS is more sensitive to lower frequency than high frequency (visual) information. [1], [7], [8], [9]. Taking this into account, the following points are relevant to digital image watermarking in the frequency domain: (i) A watermark should ideally be embedded in the higher frequency range of an image in order to achieve better perceptual invisibility but only on the understanding that high frequency components can be distorted or deleted after attacks such as lossy compression, re-sampling or scanning, for example; (ii) in order to prevent the watermark from being attacked, it is often necessary to embed it into the lower frequency region of the spectrum, which can not be attacked without compromising the image given that the HVS is more sensitive in this region; (iii) given points (i) and (ii), in order to embed a watermark in an image optimally (i.e. so that it can survive most attacks), a reasonable tradeoff is to embed a watermark into the intermediate frequency range of the image [10], [11], [12], [13].

\section{DIFFUSION AND CONFUSION BASED WATERMARKING}

We consider an approch to watermarking plaintext using both diffusion and confusion. The basic method is as follows: Given a plaintext image and a covertext image, the stegotext image is given by

$$
\text { stegotext }=\text { ciphertext }+ \text { covertext }
$$

where

$$
\text { ciphertext }=\text { cipher } \otimes \otimes \text { plaintext }
$$

and $\otimes \otimes$ denotes the two-dimensional convolution operation. The problem is to find a cipher which provides a ciphertext that, given the equation above, can be well hidden in the covertext.

\section{A. Fresnel Diffusion Watermarking}

Consider a watermarking model given by

$$
I_{3}(x, y)=r p(x, y) \otimes \otimes I_{1}(x, y)+I_{2}(x, y)
$$

with 'Fresnel' Point Spread Function (PSF)

$$
p(x, y)=\frac{1}{2}\left(1+\cos \left[\alpha\left(x^{2}+y^{2}\right)\right]\right.
$$


and where

$$
\left\|p(x, y) \otimes \otimes I_{1}(x, y)\right\|_{\infty}=1 \text { and }\left\|I_{2}(x, y)\right\|_{\infty}=1 .
$$

Here, $r$ controls the extent to which the host image $I_{2}$ dominates the diffused watermark image $I_{1}$. In effect, $r$ is like a Signal-to-Noise Ratio, or, in this application a 'Diffusion-to-Confusion' Ratio The output of this process $I_{3}$ is the watermarked host image. Recovery of the watermark image is then based on the following process:

$$
I_{1}(x, y)=\frac{1}{r} p(x, y) \odot \odot\left(I_{3}(x, y)-I_{2}(x, y)\right.
$$

where $\odot \odot$ denote two-dimensional correlation. The method is implemented numerically using a Fast Fourier Transform and application of the two-dimensional convolution and correlation theorems, i.e.

$$
p \otimes \otimes f \Longleftrightarrow P F
$$

and

$$
p \odot \odot f \Longleftrightarrow P^{*} F
$$

respectively, where $\Longleftrightarrow$ denotes transformation from 'image space' to 'Fourier space'.

Figure 1 shows an example result of implementing this watermaking method where a digital image of Albert Einstein (the covertext) is watermarked with a binary image of his most famous of equation (the plaintext). Note that the dynamic range of the diffused field and the reconstruction is relatively low and the images given in Figure 1 are displayed by re-quantisation to an 8-bit grey scale of the data $\min [I(x, y)] \leq I(x, y) \leq \max [I(x, y)]$. On the other hand, the low dynamic range of the diffused field allows the diffused field to be added to the host image without any significant distortions becoming discernable other than increasing the brightness of the image ${ }^{1}$.

Fresnel diffusion is only of value when the plaintext is of binary form (i.e. a binary image) and when the covertext is well textured throughout in order to 'hide' the diffused plaintext.

In this application, the host image together with $(\alpha, r)$ form the key where the algorithm is taken to be in the public domain. Given these conditions, the method is useful for application to watermarking digital images provided that the distortion accompanying the restoration of the watermark is acceptable. However, the method is not particularly well suited to document (hard-copy) watermarking accept under special circumstances. One such example is given in Figure 2 which illustrates a method designed whereby, using standard security printing technology, a covert digital thread can be introduced (typically into a print file) that reflects a conventional overt thread. In this example, a one-dimensional Fresnel transform (a symmetric chirp function) is used to

\footnotetext{
${ }^{1}$ In each case the data is re-normalised to floating point values between 0 and 1 before application of grey-scale quantisation.
}
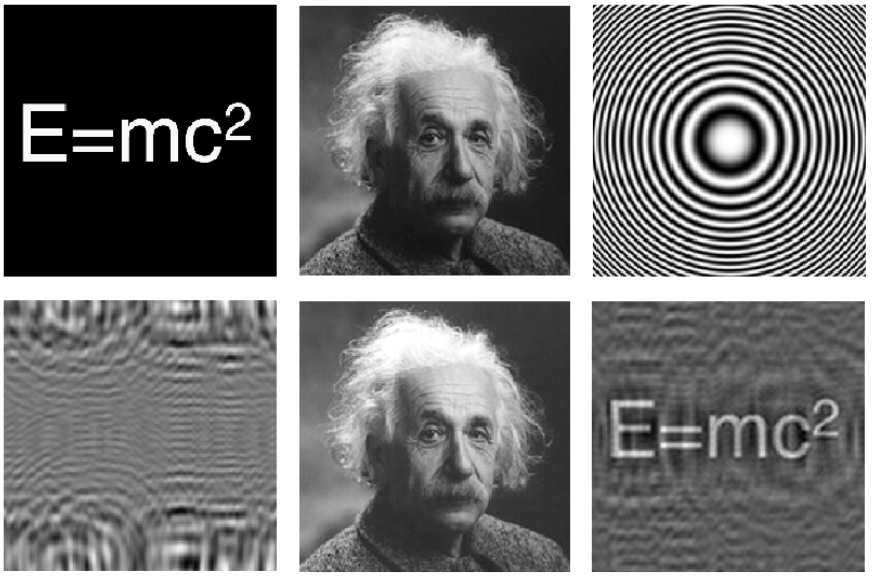

Figure 1. From top to bottom and from left to right (all images are $512 \times 512$ ): Watermark $I_{1}$, host image $I_{2}$, PSF $p$ for $\alpha=0.001$, diffused image $p \otimes \otimes I_{1}$, host image after watermarking $I_{3}$ for $r=0.1$, recovered watermark.

encode a single or multiple bar code and the result embedded into an existing print file. Recovery of the 'digital thread' is obtained through correlation of the same symmetric chirp function with a scanned image. This approach is analogous to the application of a matched filter and, in particular, the deconvolution on linear frequency modulated chirps Applications include currency, bank bonds and other security documents. In this case the 'watermarking model' is based on the following:

$$
I_{3}(x, y)=\operatorname{rp}(x, y) \otimes \otimes I_{1}(x, y)+I_{2}(x, y)
$$

where $I_{1}$ is a binary image consisting of a bar code (single or multiple bars),

$$
p(x, y)=\frac{1}{2}\left[1+\cos \left(\alpha x^{2}\right)\right]
$$

and $I_{2}$ is the host image. Recovery of the bar code (i.e. estimation $\hat{I}_{1}$ of $I_{1}$ is then given by

$$
\hat{I}_{1}(x, y) \sim p(x, y) \odot \odot I_{3}(x, y)+\epsilon
$$

where

$$
\epsilon=p(x, y) \odot \odot I_{2}(x, y)
$$

such that, provided $I_{2}$ does not correlate with $p$ (e.g. $I_{2}$ is a textured image), then

$$
\|\epsilon(x, y)\|<<\left\|p(x, y) \odot \odot I_{3}(x, y)\right\|
$$

This condition allows an exact reconstruction of $I_{1}$ to be obtained through application of a threshold to binarize $\hat{I}_{1}$.

\section{B. Noise Diffusion Watermarking}

The principal weakness associated with Fresnel diffusion is that the cipher is based on a deterministic function. 

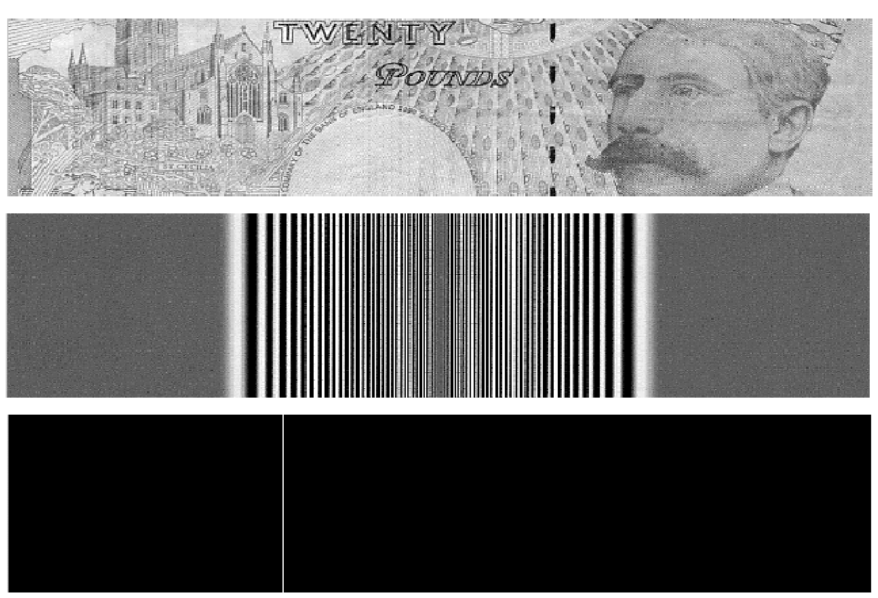

Figure 2. 600dpi scan of a 20 Pounds Sterling Bank (of England) note (above) whose graphic file includes the addition of symmetric chirp (centre) and recovery of a digital thread.

To overcome this, we consider a noise diffusion approach. Diffusion by noise is compounded in the model

$$
I(x, y)=n(x, y) \otimes \otimes I_{0}(x, y)
$$

where $n$ is some stochastic two-dimensional field and $I_{0}$ is an input image. There are two approaches to solving the problem: Given $I$ and $n$, obtain $I_{0}$. We can invert or deconvolve by using the convolution theorem giving

$$
I_{0}(x, y)=\mathcal{F}_{2}^{-1}\left[\frac{\widetilde{I}\left(k_{x}, k_{y}\right) N^{*}\left(k_{x}, k_{y}\right)}{\left|N\left(k_{x}, k_{y}\right)\right|^{2}}\right]
$$

where $\mathcal{F}_{2}^{-1}$ is the (two-diemsnional) inverse Fourier transform operator, $N$ is the Fourier transform of $n$ and $\widetilde{I}$ is the Fourier transform of $I$ (with spatial frequencies $k_{x}$ and $k_{y}$ ). However, this approach requires regularisation in order to eliminate any singularities when $|N|^{2}=0$ through application of a constrained deconvolution methods. Alternatively, if $n$ is the result of some random number generating algorithm, and since the functional form of $n$ is arbitrary, we can construct the stochastic field

$$
m(x, y)=\mathcal{F}_{2}^{-1}\left[\frac{N\left(k_{x}, k_{y}\right)}{\left|N\left(k_{x}, k_{y}\right)\right|^{2}}\right]
$$

where $\left|N\left(k_{x}, k_{y}\right)\right|^{2}>0$, the diffused field now being given by

$$
I(x, y)=m(x, y) \otimes \otimes I_{0}(x, y) .
$$

The inverse problem is then solved by correlating $I$ with $n$, since

$$
n(x, y) \odot \odot I(x, y) \Longleftrightarrow N^{*}\left(k_{x}, k_{y}\right) \widetilde{I}\left(k_{x}, k_{y}\right)
$$

and

$$
N^{*}\left(k_{x}, k_{y}\right) \widetilde{I}\left(k_{x}, k_{y}\right)=N^{*}\left(k_{x}, k_{y}\right) M\left(k_{x}, k_{y}\right) \widetilde{I}_{0}\left(k_{x}, k_{y}\right)
$$

$$
=N^{*}\left(k_{x}, k_{y}\right) \frac{N\left(k_{x}, k_{y}\right)}{\left|N\left(k_{x}, k_{y}\right)\right|^{2}} \widetilde{I}_{0}\left(k_{x}, k_{y}\right)=\widetilde{I}_{0}\left(k_{x}, k_{y}\right)
$$

so that

$$
I_{0}(x, y)=n(x, y) \odot \odot I(x, y) .
$$

The condition that $\left|N\left(k_{x}, k_{y}\right)\right|^{2}>0$ is simply achieved by implementing the following process:

$\forall k_{x}, k_{y}$

$$
\begin{aligned}
& \text { if } \quad\left|N\left(k_{x}, k_{y}\right)\right|^{2}=0 \\
& \text { then }\left|N\left(k_{x}, k_{y}\right)\right|^{2}=1
\end{aligned}
$$

This result can be used to 'embed' one image in another. Consider the case when we have two independent images $I_{1}(x, y) \geq 0 \forall x, y$ and $I_{2}(x, y) \geq 0 \forall x, y$ and we consider the case of embedding $I_{1}$ with $I_{2}$. We construct a stochastic field $m(x, y) \geq 0 \forall x$, y a priori and consider the equation

$$
I_{3}(x, y)=r m(x, y) \otimes \otimes I_{1}(x, y)+I_{2}(x, y)
$$

where

$$
\left\|m(x, y) \otimes \otimes I_{1}(x, y)\right\|_{\infty}=1 \text { and }\left\|I_{2}(x, y)\right\|_{\infty}=1 .
$$

By normalising the terms in this way, the coefficient $0 \leq$ $r \leq 1$, can be used to adjust the relative magnitudes of the terms such that the diffused image $I_{1}$ is a perturbation of the 'host image' $I_{2}$. This provides us with a way of watermarking one image with another, $r$ being Diffusionto-Confusion of 'Watermarking' Ratio. This approach is of course identical to the Fresnel diffusion method considered earlier but where the Fresnel PSF is replaced with the preconditioned stochastic field $m$. However, for applications in image watermarking, the diffusion of an image with noise provides a superior result because: (i) a stochastic field provides more uniform diffusion; (ii) noise fields can be generated using random number generators that depend on a single initial value or seed (i.e. a private key).

The noise field can be created using conventional Pseudo Random Number Generators or chaos based iteration schemes [30] for which a PIN (Personal Identity Number) or a password are required. Alternatively, noise fields can be created indirectly through application of various commercial (key dependent) cryptosystems (by encrypting a file consisting of a single plaintext character, for example) or by using genuine random number generating systems such as HotBits [31] to create $n$ (which must then be encrypted).

An example of this approach is shown in Figure 3 which should be compared with Figure 1. Here, the image $I_{2}$ (the 'host image' - covertext) is watermarked with another image $I_{1}$ (the 'watermark image' - plaintext) to produce output image $I_{3}$ with $r=0.01$. The relatively small perturbation of the term $m \otimes \otimes I_{1}$ to the host image $I_{2}$ for $r=0.01$ 

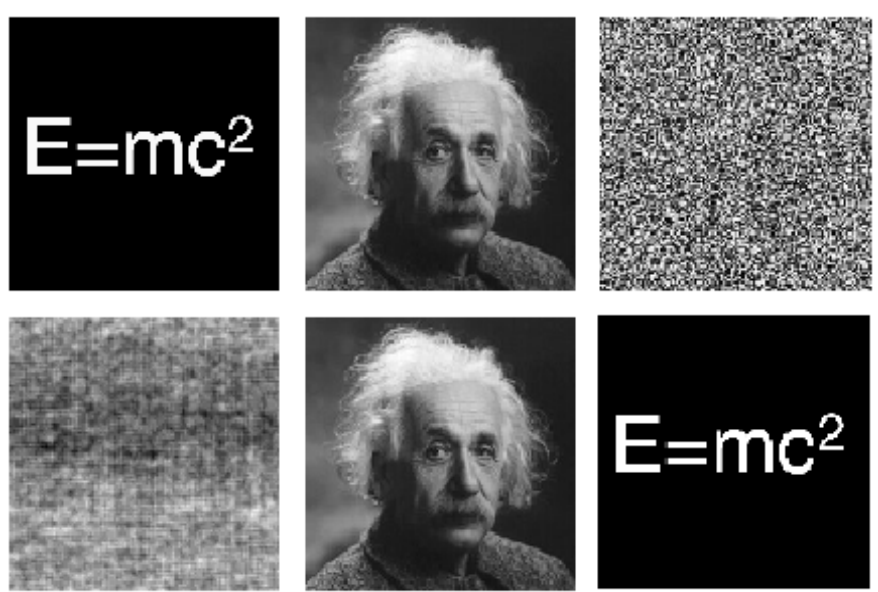

Figure 3. From top to bottom and from left to right (all images are $512 \times 512$ ): Watermark $I_{1}$, host image $I_{2}$, PSF $m$, diffused image $m \otimes \otimes I_{1}$, host image after watermarking $I_{3}$ for $r=0.01$, recovered watermark.

does not affect the output image in any way that is visually significant.

A further advantage of noise diffusion is that it is not limited to watermarking covertexts with binary image plaintexts. However, the effect of adding the diffused greyscale or colour watermark image to the host image yields a different, slightly brighter image because of the perturbation of $I_{2}$ by $r m \otimes \otimes I_{1}$. This effect can be minimized by introducing a smaller Watermarking Ratio such that the perturbation is still recoverable by subtracting the host image from the watermarked image, an example being given in Figure 4.
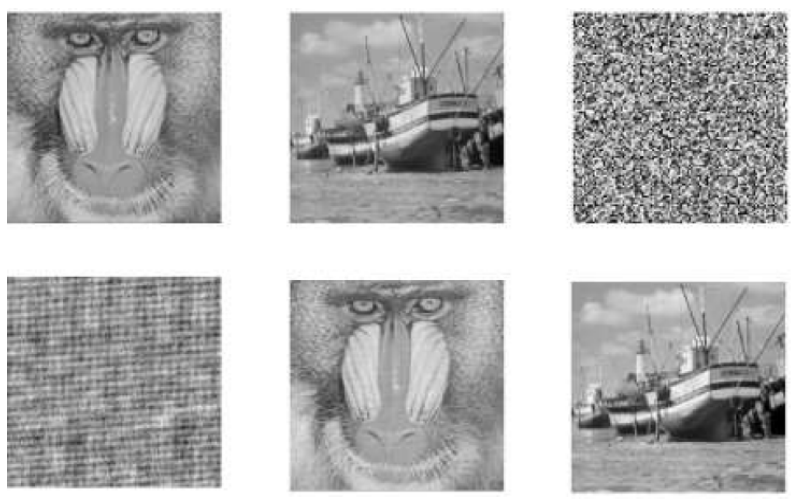

Figure 4. Example of watermarking an image with another image using noise based diffusion. The 'host image' $I_{2}$ (top-left ) is watermarked with the 'watermark image' $I_{1}$ (top-centre) using the diffuser (top-right) given by a uniform noise field $n$ whose pixel-by-pixel values depend upon the seed used (the private key). The result of computing $m \otimes \otimes I_{1}$ (bottom-left) is added to the host image for $r=0.1$ to generate the watermarked image $u$ (bottom-centre). Recovery of the watermark image $I_{1}$ (bottom-right) is accomplished by subtracting the host image from the watermarked image and correlating the result with the noise field $n$.

\section{HARDCOPY STEGANOGRAPHY}

The model

$$
\text { stegotext }=\text { ciphertext }+ \text { covertext }
$$

can be applied for watermarking digital images associated with electronic-to-electronic type communications in which there is no or minimal loss of information. This method can be used to watermark digital images for the purpose of authentication but can also be viewed as a method of covertly transmitting ciphertext when the plaintext is converted to the form of a digital image. Steganography and watermarking techniques are also of value for hardcopy 'data' which has a range of applications for authenticating printed material and copyright validation, for example. However, to be of practical value to the security printing industry the methods must be robust to the significant distortions generated by the printing and/or scanning process. A simple approach is to add information to a printed page that is difficult to see. For example, some modern colour laser printers, including those manufactured by HP and Xerox, print tiny yellow dots which are added to each page. The dots are barely visible and contain encoded printer serial numbers, date and time stamps. This facility provides a useful forensics tool for tracking the origins of a printed document which has only relatively recently been disclosed.

\section{A. Diffusion Only Watermarking}

If a stegotext image is printed and scanned back into electronic form, then the print/scan process will yield an array of pixels that will be significantly different from the original electronic image even though it might 'look' the same. These differences can include the size of the image, its orientation, brightness, contrast and so on. Of all the processes involved in the recovery of the watermark, the subtraction of the host image from the watermarked image is critical. If this process is not accurate on a pixel-by-pixel basis and deregistered for any of many reasons, then recovery of the watermark by correlation will not be effective. However, if we make use of the diffusion process alone, then the watermark can be recovered via a print/scan because of the compatibility of the processes involved. However, in this case, the 'watermark' is not covert but overt.

Depending on the printing process applied, a number of distortions will occur which diffuse the information being printed. Thus, in general, we can consider the printing process to introduce an effect that can be represented by the convolution equation

$$
I_{\text {print }}=p_{\text {print }} \otimes \otimes I .
$$

where $I$ is the original electronic form of a diffused image (i.e. $I=m \otimes \otimes I_{0}$ ) and $p_{\text {print }}$ is the point spread function of the printer. An incoherent image of the data, obtained using a flat bed scanner, for example (or any other incoherent optical 
imaging system), will also have a characteristic point spread function $p_{\text {scan }}$ say. Thus, we can consider a scanned image to be given by

$$
I_{\text {scan }}=p_{\text {scan }} \otimes \otimes I_{\text {print }}
$$

where $I_{\text {scan }}$ is taken to be the digital image obtained from the scan. Now, because convolution is commutative, we can write

$I_{\text {scan }}=p_{\text {scan }} \otimes \otimes p_{\text {print }} \otimes \otimes p \otimes \otimes I_{0}=p \otimes \otimes p_{\text {scan } / \text { print }} \otimes \otimes I_{0}$

where

$$
p_{\text {scan } / \text { print }}=p_{\text {scan }} \otimes \otimes p_{\text {print }}
$$

which is the print/scan point spread function associated with the processing cycle of printing the image and then scanning it back into electronic form. By applying the method discussed earlier, we can obtain a reconstruction of the watermark whose fidelity is determined by the scan/print PSF. However, in practice, the scanned image needs to be re-sized to that of the original. This is due to the scaling relationship (for a function $f$ with Fourier transform $F$ )

$$
f(\alpha x, \beta y) \Longleftrightarrow \frac{1}{\alpha \beta} F\left(\frac{k_{x}}{\alpha}, \frac{k_{y}}{\beta}\right) .
$$

The size of any image captured by a scanner or other device will depend on the resolution used. The size of the image obtained will inevitably be different from the original because of the resolution and window size used to print the diffused image $I$ and the resolution used to scan the image. Since scaling in the spatial domain causes inverse scaling in the Fourier domain, the scaling effect must be 'inverted' before the watermark can be recovered by correlation since correlation is not a scale invariant process. Re-sizing the image (using an appropriate interpolation scheme such as the bi-cubic method, for example) requires a set of two numbers $a$ and $b$ (i.e. the $a \times b$ array used to generate the noise field and execute the diffusion process) that, along with the seed required to regenerate the noise field $n$, provides the 'private keys' needed to recover the data from the diffused image. An example of this approach is given in Figure 5 which shows the result of reconstructing four different images (a photograph, finger-print, signature and text) used in the design of an impersonalized bank card. The use of 'diffusion only' watermarking for print security can be undertaken in colour by applying exactly the same diffusion/reconstruction methods to the red, green and blue components independently. This provides two additional advantages: (i) the effect of using colour tends to yield better quality reconstructions because of the colour combination process; (ii) for each colour component, it is possible to apply a noise field with a different seed. In this case, three keys are required to recover the watermark although it should be noted that, due to the errors associated in the extraction of each colour component from a colour scan, this approach

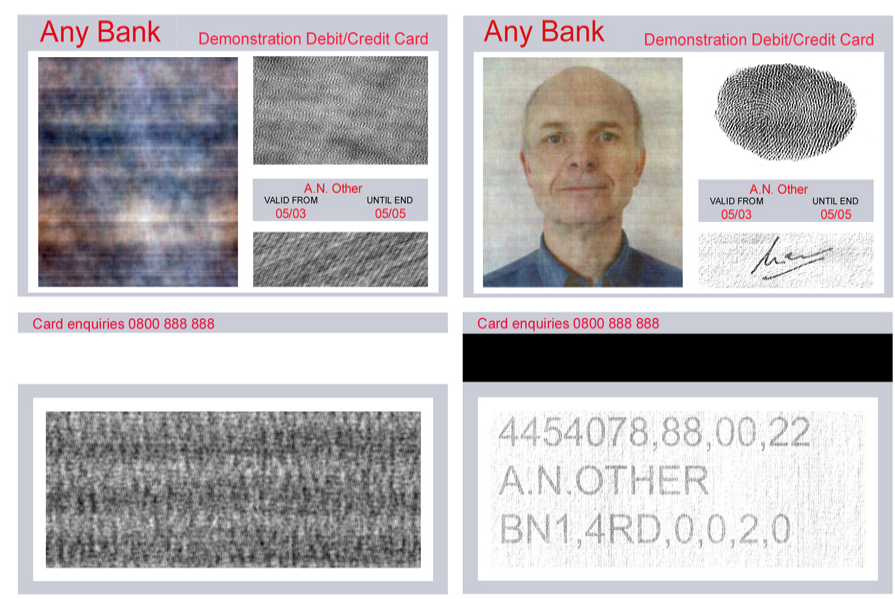

Figure 5. Example of the application of 'diffusion only' watermarking. In this example, four images of a face, finger-print, signature and text have been diffused using the same cipher and printed on the front (top-left) and back (bottom-left) of an impersonalized identity card using a $600 \mathrm{dpi}$ printer. The reconstructions (top-right and bottom-right, respectively) are obtained using a conventional flat-bed scanner based on a 300 dpi greylevel scan.

does not yield reconstructions with the same degree of robustness as in the case when the same key/algorithm is used for each colour component.

Because this method is based on convolution alone and since

$$
I_{\text {scan }}=p_{\text {scan } / \text { print }} \otimes \otimes I_{0}
$$

as discussed earlier, the recovery of the $I_{0}$ will not be negated by the distortion of the PSF associated with the print/scan process, just limited or otherwise by its characteristics. Thus, if an image is obtained of the printed data field $p \otimes \otimes I_{0}$ which is out of focus due to the characteristics of $p_{\text {scan/print }}$, then the reconstruction of $I_{0}$ will be out of focus to the same degree. Decryption of images with this characteristic is only possible using an encryption scheme that is based a diffusion only approach. However, if a covertext image $I_{\mathrm{c}}$ is introduced so that

$$
I_{\text {scan }}=p_{\text {scan } / \text { print }} \otimes \otimes I_{0}+p_{\text {scan } / \text { print }} \otimes \otimes I_{\mathrm{c}}
$$

then because

$$
I_{\text {scan }}-I_{\mathrm{c}} \neq p_{\text {scan } / \text { print }} \otimes \otimes I_{0}
$$

recovery of the plaintext is not possible which is why we resort to a diffusion only method approach.

Figure 6 illustrates the recovery of a diffused image printed onto a personal identity card obtained captured using a mobile phone camera. In the latter case, the reconstruction is not in focus because of the wide-field nature of the lens used. However, the fact that recovery of the watermark is possible with a mobile phone means that the scrambled data can be transmitted securely and the card holders image (as in this example) recovered remotely and transmitted back to the 


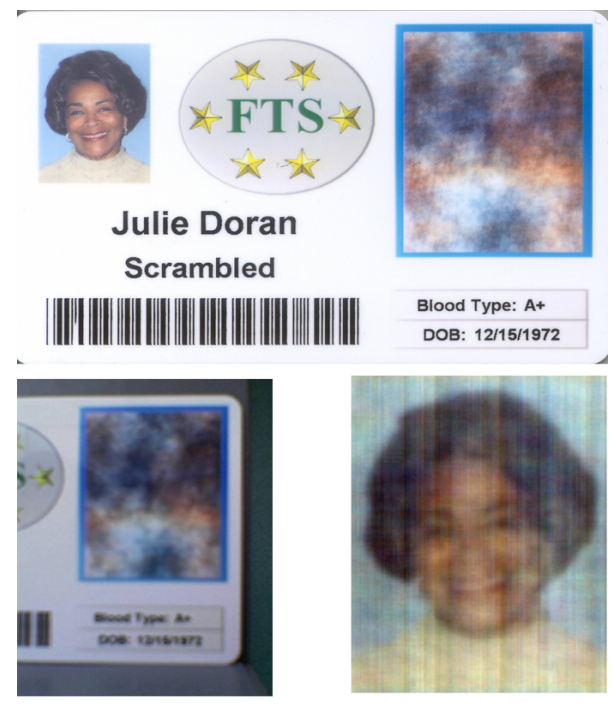

Figure 6. Example of a security card designed to include a texture code of the holders portrate (top). The images (i.e. portrate and texture code) have been printed onto the identity card at 600dpi. An image of this card (bottom-left) has been generated using a mobile phone. After cropping the texture code obtained from this low resolution data, a reconstruction can still be obtained as shown (bottom-right).

same phone for authentication. This provides the necessary physical security needed to implement such a scheme in practice and means that specialist image capture devices are not required on site. Applications of this technique to a mobile security spot check environment are clearly possible.

The diffusion process can be carried out using a variety of different noise fields other than the uniform noise field considered here. Changing the noise field can be of value in two respects: first, it allows a system to be designed that, in addition to specific keys, is based on specific algorithms which must be known a priori. These algorithms can be based on different pseudo uniform random number generators and/or different pseudo chaotic number generators that are post-processed to provide a uniform distribution of numbers. Second, the diffusion field depends on both the characteristics of the watermark image and the noise field. By utilizing different noise fields (e.g. Gaussian noise, Poisson noise), the texture of the output field can be changed. The use of different noise fields is of value when different textures are required that are aesthetically pleasing and can be used to create a background that is printed over the entire document - texture maps. In this sense, variable noise based diffusion fields can be used to replace complex print security features with the added advantage that, by de-diffusing them, information can be recovered. Further, these fields are very robust to data degradation created by soiling, for example. In the case of binary watermark images, data redundancy allows reconstructions to be generated from a binary output,
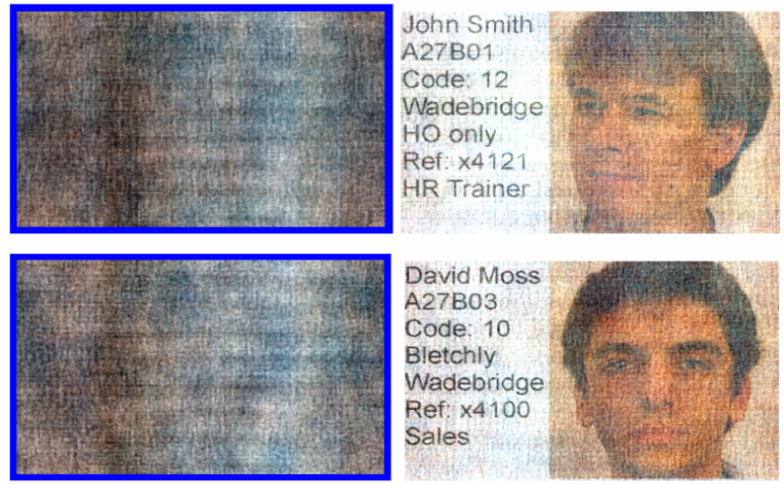

Figure 7. Example of the diffusion of composite images with the inclusion of a reference frame for enhancing and automating the processes of copping and orientation. In each case the data fields have been printed and scanned at 300 dpi.

i.e. after binarizing the diffusion field (with a threshold of $50 \%$ for example). This allows the output to be transmitted in a form that can tolerate low resolution and low contrast copying, e.g. a fax.

The tolerance of this method to printing and scanning is excellent provided the output is cropped accurately (to within a few pixels) and oriented correctly. The processes of cropping and orientation can be enhanced and automated by providing a reference frame in which the diffused image is inserted. This is illustrated in Figure 7 which, in addition shows the effect of diffusing a combination of images. This has the effect of producing a diffused field that is very similar but nevertheless conveys entirely different information. Details of the robustness of the method to various 'attacks' are provided in the Appendix.

\section{Case Study: Passport Authentication}

Like any other security document, ID card and so on, a passport consists of a number of security features depending on the sophistication of the design associated with the authority responsible for an issue. These range from the use of printing complex background, micro-printing, conventional paper watermarking, UV watermarking, foil holograms, ghost images and so on. Each of these security features may be more or less difficult to counterfeit depending on the sophistication of the feature and the counterfeiter. In this case study, we consider the use of texture coding within the context of authenticating a passport including the protocol associated with a typical 'cycle'. The method is simple and cost effective to implement in terms of the hardware required, i.e. Standard PC, flatbed scanner and printer, all of which are COTS. All that is required is a remote web site hub to which digital scans of the texture code can be emailed and where a decrypt can take place, forwarding the result back to the point of enquiry. 

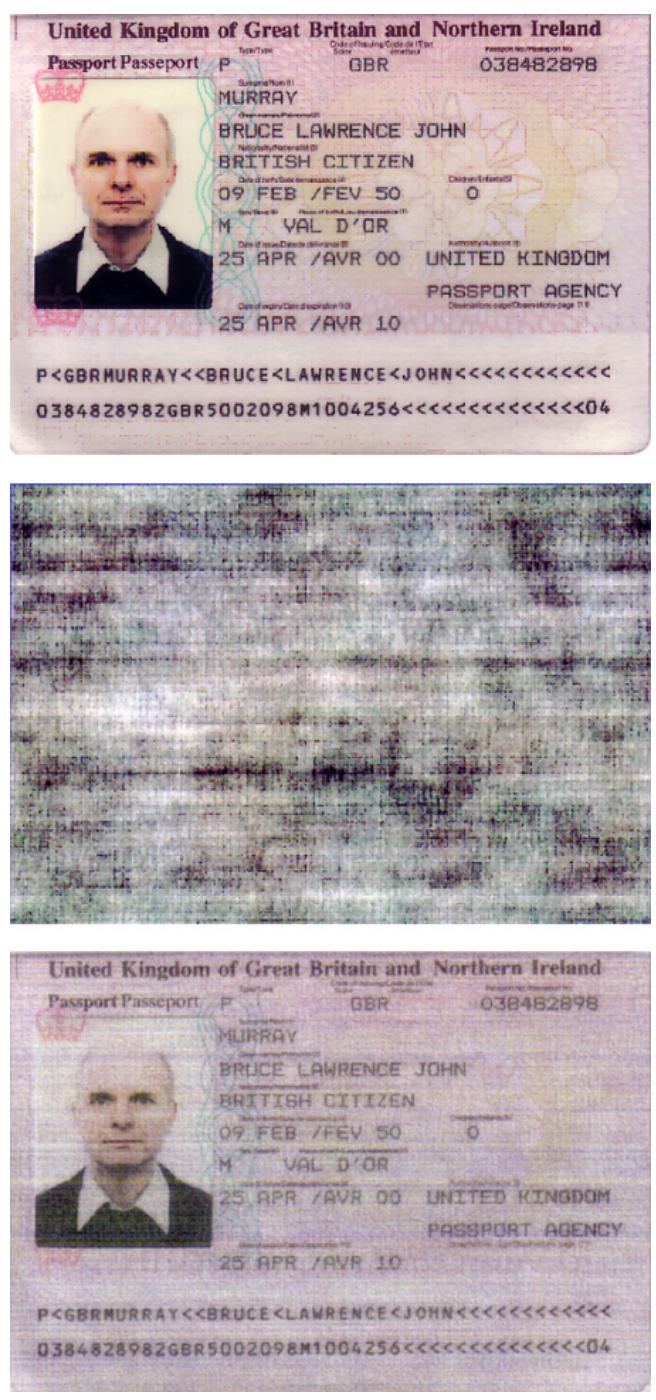

Figure 8. Example of the stochastic diffusion method applied to passport authentication: Original image scanned from a passport at 400dpi (above), printed image after applying stochastic diffusion (centre) and reconstruction after scanning the printed stochastic field at 300dpi (below).

The principal idea is to take a low resolution scan (say 600dpi) of the page (or pages) of a passport that contains the primary information, e.g. Passport number, Name, Date of birth, Signature and Photograph of the passport holder - the plaintext. This plaintext is then forwarded to a designated Hub where it is diffused with a unique noise field that is maintained at the Hub alone to produce the ciphertext. The result is then emailed back to the user, printed and the result (permanently or as required) inserted into the passport, a process that is similar to issuing a Visa, for example. At any point of contact, if the passport requires authentication, the ciphertext is scanned and the digital image emailed to the appropriate Hub where upon it is decrypted and the result (the watermark) sent back to the point of origin. Automation of this cycle would require a new infrastructure to be established which is both time consuming and expensive. Instead, the cycle described above would be best suited for use with regard to spot checks at an airport terminal, for example, especially if the holder of the passport or the passport itself is suspect. The scanning process (using a standard flat bed scanner, for example) can then be undertaken while the holder of the passport is waiting for it to be authenticated (or otherwise) based on a visual comparison between the decrypt and the plaintext.

Figure 8 shows an example of the technique applied to a composite image scanned from a passport, an application which is cheap and simple to implement with regard to authenticating a passport holders personal information. The degradation associated with the reconstruction is due to the low resolution of the printing and scanning rather than the information hiding method. Unless the correct stochastic field is used (as determined by the keys), it is not possible to reconstruct the image making counterfeiting or forgery improbable. In this case the scan has been emailed as a JPEG attachment where decryption can take place remotely.

\section{DISCUSSION}

Valuable paper documents are subject to misuse by criminals. This is largely due to the dramatic improvement in personal computer hardware and peripheral equipment. Embedding watermarks into a printed document is one way to secure them. The ability to extract the watermark from a printed copy is generally useful to help establish ownership, authenticity, and to establish the origin of an unauthorized disclosure. However, finding a robust watermarking technique is a continuing challenge. This is due to extensive amount of noise that is added when a document is printed and scanned. Moreover, printed documents do not maintain their quality over time.

In this paper, we have presented a robust watermarking method for paper security. Unlike traditional watermarking techniques, this approach can extract the hidden watermark after a print/scan attack which is achieved by using the convolution and correlation processes for coding and decoding respectively. This approach is chosen because of its compatibility with the principles of the physical optics involved in scanning a document. The watermark $w$ is diffused (convolved) with a noise field $n$ and placed into the background of a covertext, typically a text document. The watermark can be recovered by removing the covertext $f$ using a modified median filter. We then correlate the diffused watermark with the original noise field. This process (i.e. coding and decoding) is compounded in the following 
formula:

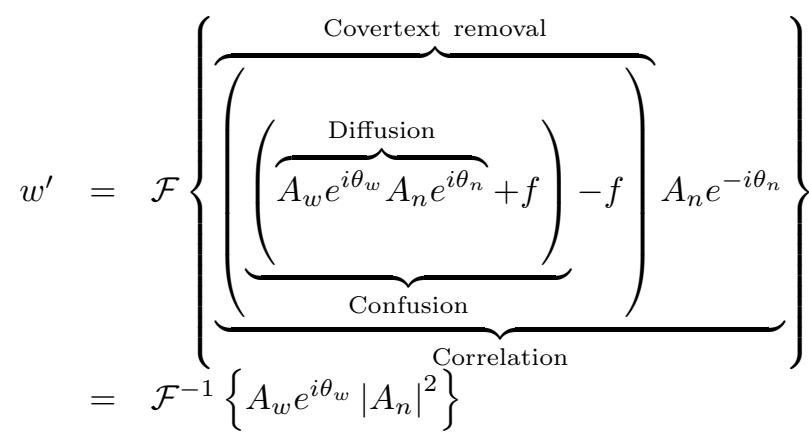

where $w^{\prime}$ is the extracted watermark, $A_{w}$ and $A_{n}$ are the amplitude spectra of the watermark and cipher respectively, $\theta_{w}$ and $\theta_{n}$ are the respective phase spectra. The extracted watermark is a noisy version of the embedded watermark. This noise is due to the power spectrum $\left|A_{n}\right|^{2}$. In order to enhance the extracted watermark, we have to eliminate the power spectrum term or at least minimize its effect. One way to do this is to divide the output over the power spectrum during the diffusion step or correlation step. To avoid singularities, we replace each and all zeros that occur in $\left|A_{n}\right|^{2}$ by 1 . Alternatively, we can choose $n$ such that it has a homogeneously distributed power spectrum across all frequencies, (such as white noise) or pre-process $n$ by replacing it's amplitude spectrum with a constant value. However, these conditions are restrictive and the regularisation method discussed above to avoid singularities is both simple and effective.

The method is robust to a wide variety of attacks including geometric attacks, drawing, crumpling and print/scan attacks as discussed in the Appendix. Further the method is relatively insensitive to lossy compression, filtering, amplitude adjustments, additive noise and thresholding. The principal weakness of the system is its sensitivity to rotation and cropping. This can be minimized by orienting the document correctly and accurately before scanning and using automatic cropping software which is available with selected scanners (e.g. Cannon scanners). Alternatively, introduction of a frame provides a reference feature from which an accurate crop can be obtained.

The visibility of the diffused watermark and the compatibility of this system with the physical principles of an imaging system, increase the robustness of the system and provides a successful approach to the extraction of the watermark after scanning at low resolution. Moreover, using correlation in the extraction phase increases the robustness of the system to some important attacks such as translation and cropping (most likely to occur during a scan).

The system is secure in that it can not be attacked easily. First, the feature is not 'suspicious' as many documents have a background texture. Second, the attacker does not know the algorithm used to generate the diffused watermark. Even if the attacker does know the algorithm, he/she must still know a significant amount of information before the system can be broken, such as: the correct key, the diffusion operator type, the original image size and so on.

For interested readers, a prototype system is available for trial purposes which can be downloaded from http://eleceng.dit.ie/arg/downloads/Document_Authentication.zip

\section{ACKNOWLEDGMENTS}

J M Blackledge is supported by the Science Foundation Ireland (Stokes Professorship Programme).

\section{REFERENCES}

[1] C. T. Hsu and J. Ling, Hidden digital watermarks in images, IEEE Transactions on Image Processing, Vol. 8, 58-68, 1999.

[2] J. Zaho and E. Koch, Embedding robust labels into images for copyright protection, Proceedings of the International Conference on Intellectual Property Rights for Information, Knowledge and New Techniques, 242-251, (München, Wien: Oldenbourg Verlag), 1995.

[3] I. J. Cox, J. Kilian, T. Leighton, and T. Shamoon, A secure, robust watermark for multimedia, First International Workshop on Information Hiding, Ed. R. Anderson, 1174 of Lecture Notes in Computer Science, 183-206, SpringerVerlag, 1996.

[4] M. Barni, F. Bartolini, V. Cappellini, and A.Piva, A DCTdomain system for robust image watermarking, Signal Processing (EURASIP), Vol. 66, 357-372, 1998.

[5] J. J. K. O. Ruanaidh, W. J. Dowling, and F. M. Boland, Watermarking digital images for copyright protection, IEE Proceedings on Vision, Signal and Image Processing, Vol. 143, 250-256, 1996.

[6] M. D. Swanson, B.Zhu, and A. H. Tewfik, Transparent robust image watermarking, International Conference on Image Processing, IEEE,3, 211-214, 1996.

[7] J. J. Chae and B. Manjunath, A technique for image data hiding and reconstruction without host image, Proc. Of SPIE Electronic Imaging'99, Security and Watermarking of Multimedia Contents, Wong and Delp, eds., 3657, (San Jose, California), January 1999.

[8] A. Bors and I. Pitas, Image watermarking using block site selection and d.c.t. domain constraints, Optics Express 3, 512-523, Dec 1998.

[9] B. Tao and B. Dickenson, Adaptive watermarking in the DCT domain, International Conference on Acoustics and Signal Processing, 1997.

[10] R. C. Gonzalez and R. E. Woods, Digital Image Processing, Prentice Hall, New Jersey, 2nd ed., 2002.

[11] X. Xia, C. Boncelet, and G. Arce, A multi-resolution watermark for digital Images, in Proc. IEEE Int. Conf. On Image Processing, 1, 548-551, Oct 1997. 
[12] A. Lumini and D. Maio, A wavelet-based image watermarking scheme, Proceedings of the IEEE International Conference on Information Technology: Coding and Computing, 122-127, March 2000.

[13] D. Kundur and D. Hatzinakos, Digital watermarking using multi-resolution wavelet decomposition, Proceeding of the IEEE International Conference on Acoustics, Speech and Signal Processing, 6, 2969-2972, 1998.

[14] D. Kundur and D. Hatzinakos, A robust digital image watermarking method using wavelet-based fusion, International Conference on Image Processing, IEEE, 544-547, (Santa Barbara, California, U.S.A.), Oct 1997.

[15] M. D. Levine, Vision in Man and Machine, McGraw-Hill, Toronto, 1985.

[16] J. Ohnishi and K. Matsui, Embedding a seal into a picture under orthogonal wavelet transform, Proc. Int. Conference on Multimedia Computing and Systems, 514-521, June 1996.

[17] M. Barni, F.Bartolini, V.Cappellini, A. Lippi, and A. Piva, A DWT-based technique for spatio-frequency masking of digital signatures, Proc. Of SPIE Electronic Imaging'99, Security and Watermarking of Multimedia Contents, 3657, 31-39, (San Jose, California), January 1999.

[18] A. S. Lewis and G. Knowles, Image compression using the 2-d wavelet transform, IEEE trans. Image Processing, 1, 240-250, April 1992.

[19] H. Inoue, T. Katsura, A. Miyazaki, and A. Yamamoto, A digital watermark technique based on the wavelet transform and its robustness on image compression and transformation, IEICE Transactions on Fundamentals of Electronics, E82-A, 2-10, Jan 1999.

[20] J. Shapiro, Embedded image coding using zerotrees of wavelet coefficients, IEEE Trans. Signal Processing, 41(12), 3445-3462, 1993.

[21] J. J. K. O. Ruanaidh and T. Pun, Rotation, scale and translation invariant spread spectrum digital image watermarking, Signal Processing(EURASIP), 66, 303-317, May 1998.

[22] J. J. K. O. Ruanaidh, W. J. Dowling, and F. M. Boland, Phase watermarking of digital images, Proceedings of the IEEE International Conference on Image Processing, 3, 239242, sep 1996.

[23] V. Solachidis and I. Pitas, Circularly symmetric watermark embedding in 2-d DFT domain, International Conference on Acoustics, Speech and Signal Processing, IEEE Signal Processing Society, 1563-1565, (Phoenix, Arizona, USA), March 1999.

[24] W. Kim, J. Lee, and W. Lee, An image watermarking scheme with hidden signature, IEEE Proceeding of the International Conference on Image Processing, 206-210, (Japan), Oct 1999.

[25] H. Raymound, F. Chan, and K. Yeung, A frequency domain watermarking scheme, Jan 2001.
[26] A. Herrigel, J. O'Ruanaidh, H. Petersen, and S. Pererira, Secure copyright protection techniques for digital images, Information Hiding of Lecture Notes in Computer Science, D. Aucsmith, ed., 1525, 169-190, Springer-Verlag, 1998.

[27] C.-Y. Lin, M. Wu, J. Bloom, I. Cox, M. Miller, and Y. Lui, Rotation, scale, and translation resilient public watermarking for images, Security and Watermarking of Multimedia Contents II, Proceedings of SPIE, P. W. Wong and J. E. Delp, Eds., 3971, 90-98, 2000.

[28] M. Kankanhalli and R. Ramakrishnan, Content based watermarking of images, 6th ACM International Multimedia Conference, 61-70, (Bristol, England), Sep 1998.

[29] R. J. Anderson and F. Petitcolas, On the limits of steganography, IEEE: Selected Areas in Communications, 16, 474481, May 1998.

[30] J. M. Blackledge, Multi-algorithmic Cryptography using Deterministic Chaos with Applications to Mobile Communications, ISAST Transactions on Electronics and Signal Processing (ISSN 1797-2329), 1(2), 23 - 64, 2008

[31] http://www.fourmilab.ch/hotbits/

[32] M. Kutter and F. Hartung, Introduction to watermarking techniques, Information Hiding: Techniques for Stegangraphy and Difgital Watermarking, S. Katezenbeisser and F. A. P. Petitcolas, Eds., Ch. 5, 97-120, Artech House, Boston, 2000.

[33] A. K. Jain, S. Pankanti, and R. Bolle, eds., BIOMETRICS: Personal Identification in Networked Society, Kluwer, 1999.

[34] A. K. Jain, F. D. Griess, and S. D. Connell, On-line signature verification, Pattern Recognition 35, 2963-2972, December 2002.

[35] I. J. Cox, M. Miller, and J. Bloom, Digital Watermarking, Morgan Kaufmann, 2002.

[36] J.-L. Dugelay and F. A. P. Petitcolas, Possible counterattack against random geometric distortions, Security and Watermarking of Multimedia Content, 3971, 338-345, SPIE, 2000 .

[37] S. Pereira, J. K. O. Ruanaidh, and T. Pun, Secure robust digital watermarking using the lapped orthogonal transform, Security and Watermarking of Multimedia Content, 3657, 21-30, SPIE, 1999.

[38] J. J. K. O. Ruanaidh and T. Pun, Rotation, scale and translation invariant spread spectrum digital image watermarking, Signal Processing(EURASIP), 66, 303-317.

[39] K. Mahmoud, Novel Methods for Print Security and Anticounterfeiting Technology $\mathrm{PhD}$ Thesis, Loughborough University, 2006. 